\title{
Absceso prostático por Sthapilococo aureus y empiema subdural: presentación de un caso
}

\author{
Cabrera Meirás F, Sanchís Bonet A, Blanco Carballo O, Martín Parada A, Duque Ruiz G, \\ Leiva Galvis O.
}

Servicio de Urología. Hospital Universitario 12 de Octubre. Madrid.

Actas Urol Esp. 2007;31(5):548-552

\section{RESUMEN}

ABSCESO PROSTÁTICO POR STHAPILOCOCO AUREUS Y EMPIEMA SUBDURAL: PRESENTACIÓN

DE UN CASO

Introducción y objetivo: Presentamos el caso de un paciente con absceso prostático y empiema subdural por Staphilococo aureus.

Material y método: Descripción de un caso de un paciente de 51 años de edad diagnosticado de absceso prostático y empiema subdural por Staphilococo aureus. Utilizamos como método de aproximación diagnóstica la sospecha clínica y la exploración física mediante tacto rectal. Como métodos de confirmación diagnóstica, pruebas de imagen, como la tomografía axial computerizada y la ecografía transrectal, que permite además el drenaje del material purulento.

Resultados: El cuadro se resolvió con ecografía transrectal y punción-drenaje de la colección y con tratamiento conservador en base a antibioterapia y derivación urinaria.

Conclusiones: El absceso prostático es en la actualidad una patología poco frecuente. Dada la gran variedad de presentación de esta entidad, hay que tener un alto grado de sospecha para su diagnóstico y una vez realizado comenzar un tratamiento inmediato agresivo. La ecografía transrectal permite, no sólo el diagnóstico, sino también la punción-drenaje del contenido purulento. El cultivo de las muestras obtenidas identifica el agente causante y la antibioterapia más adecuada.

Palabras clave: Absceso prostático. Ecografia transrectal. Staphilococo aureus. Empiema subdural.

\section{ABSTRACT}

STHAPYLOCOCCUS AUREUS PROSTATIC ABSCESS AND SUBDURAL EMPYEMA: A CASE REPORT.

Introduction and objectives: To report one case of prostatic abscess and subdural empyema by Staphylococcus aureus.

Methods: We describe the case of a 51 year old male patient who was diagnosed of prostatic abscess and subdural empyema by Staphilococcus aureus. We use clinical presentation and physical exploration based on rectal digital examination, as diagnostic approach method. And computerized axial tomography and transrectal ultrasonography, which allows the guided needle drainage of the abscess, as diagnostic confirmation methods.

Results: The clinical picture resolved with the transrectal ultrasonography guided needle aspiration of the abscess and conservative treatment with antibiotics and urinary diversion.

Conclusions: Prostatic abscess is an uncommon entity nowadays. Provided the great variety of symptoms, a great degree of clinical suspicion is needed for the diagnosis, and once it is got it, immediate aggressive treatment must be initiated. Transrectal ultrasonography allows not only the diagnosis, but also the drainage of the abscess. The culture of the obtained material identifies the etiological agent and the most specific antibiotic therapy.

Keywords: Prostatic abscess. Transrectal ultrasonography. Sthaphylococcus aureus. Subdural empyema.

Abreviaturas: TAC: Tomografia axial computerizada. RMN: Resonancia magnética nuclear. ECTR: Ecografia transrectal. ADVP: Adictos a drogas por vía parenteral. RTUP: Resección transuretral de próstata. 
$\mathrm{E}$ absceso prostático es una entidad poco frecuente que se diagnostica en el $0.2 \%$ de los pacientes con clínica urológica ${ }^{1}$. La incidencia, morbilidad y mortalidad asociada han disminuido desde la utilización de antibióticos con amplia penetración en el tejido prostático. La generalización en el uso de estos antibióticos y los cambios sociosanitarios de la población, han modificado el espectro de los microorganismos causantes de esta patología. Los bacilos Gram negativos y Staphilococo aureus han sustituido a Neisseria gonorrhoeae, el agente causal, más frecuente hasta la primera mitad del siglo $\mathrm{XX}^{2-4}$.

Es necesario recordar por otro lado, la dificultad del diagnóstico exclusivamente en base a la historia clínica y la exploración física. Es por esto que las técnicas de imagen han cosechado una amplia aceptación en los últimos años, en el diagnóstico del absceso prostático. Entre ellas destaca la ecografia transrectal, ya que además de confirmar el diagnóstico permite el tratamiento en el mismo acto ${ }^{5-7}$.

\section{CASO CLÍNICo}

Varón de 59 años de edad con los antecedentes personales de etilismo crónico y hábito tabáquico de años de evolución, que como complicaciones había presentado un episodio de hemorragia digestiva alta secundaria a analgésicos antiinflamatorios. Como antecedentes urológicos un síndrome prostático de dos años de evolución sin complicaciones secundarias.

Acude a urgencias por sindrome febril intermitente de cuatro días de evolución, al que asocia incontinencia urinaria en las últimas 48 horas, junto con disuria y dolor sacro que se irradia a ambos miembros inferiores y que empeora con los movimientos. Se asocia también tenesmo rectal de reciente comienzo. A su llegada a Urgencias se objetiva fiebre de hasta $39^{\circ} \mathrm{C}$, el paciente se encuentra hemodinámicamente estable, confuso, somnoliento y sudoroso.

A la exploración física, abdomen globuloso, blando y depresible, no peritonítico. Doloroso a la palpación en área lumbar bilateral a la altura de L5-S1, así como en área hipogástrica y región perineal. No impresiona de globo vesical. La exploración genital es normal, y el tacto rectal volumen I adenomatosa, ligeramente doloroso. La exploración neurológica muestra disminución de la fuerza en ambos miembros inferiores en su tercio superior y la sensibilidad esta conservada. Los reflejos rotuliano y aquileo están presentes.

El paciente presenta en analítica urgente con hemograma y bioquímica: Hemoglobina 14,2 g/dl, Hematocrito 41,5\%, Leucocitos 20,300 (Neutrófilos 72,9\%), Glucosa $212 \mathrm{mg} / \mathrm{dl}$, Creatinina 1,11 $\mathrm{mg} / \mathrm{dl}$, Sodio $135 \mathrm{mEq} / 1$, Potasio 3,8 mEq/1, Velocidad sedimentación $100 \mathrm{~mm} / \mathrm{h}$. Un análisis de orina con leucocitos negativos, nitritos negativos y sedimento normal. Se completa el análisis con un estudio de coagulación que determina una actividad de protrombina del $83 \%$, un tiempo de cefalina de 27,2 sg, y fibrinógeno de $574 \mathrm{mg} / \mathrm{dl}$.

Se solicitó de entrada una TAC abdomino-pélvica urgente que informaba de riñones con múltiples quistes simples y litiasis renal izquierda. No dilatación de la vía urinaria. Próstata aumentada de tamaño, observándose dos lesiones hipodensas en ambos lóbulos laterales de $3.3 \mathrm{~cm}$ y 3 cm compatibles con absceso prostático (Fig. 1).

Se instaura entonces tratamiento antibiótico empírico con Ceftriaxona $1 \mathrm{~g} / 12 \mathrm{~h}$, Ampicilina 1 g/6h y Gentamicina $240 \mathrm{mg} / 24 \mathrm{~h}$.

Con el diagnóstico de absceso prostático decidimos drenaje de la colección bajo control con ECTR y derivación urinaria.

En quirófano, se realiza punción lumbar previa a la anestesia intradural. Se observa salida de líquido cefalorraquídeo turbio-amarillento y se envía una muestra a microbiología y bioquímica, se aislaron 1070 leucocitos (85\% polimorfonucleares).

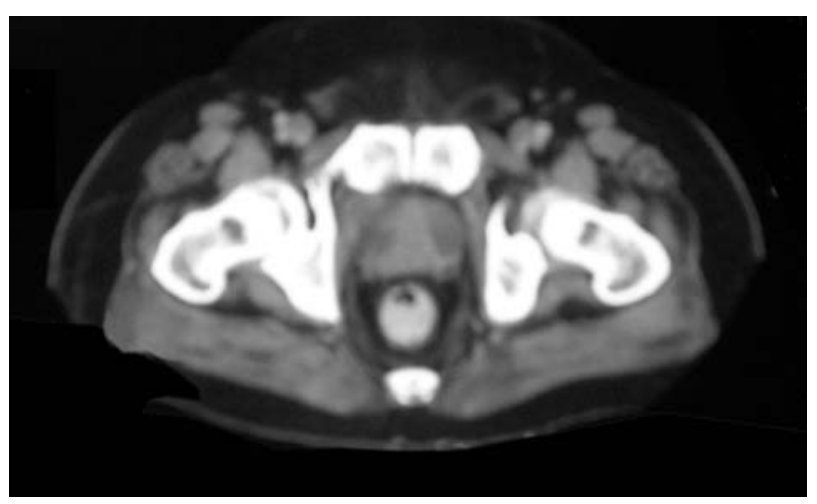

FIGURA 1: Imagen de TAC abdomino-pelvica en la que se observa una próstata aumentada de tamaño con dos áreas hipodensas en ambos lóbulos prostáticos. 
Por otro lado, se confirma el diagnóstico de absceso prostático en la ECTR, en la que se evidencian, dos colecciones de 30 y $28 \mathrm{~mm}$ que afectan al lóbulo izquierdo y derecho respectivamente, siendo el resto de la próstata heterogénea sin claras áreas abscesificadas (Fig. 2). Mediante punción-drenaje vía perineal evacuamos 10cc de pus, de las colecciones descritas, dejando colocado una sonda de cistostomía a modo de drenaje. Derivamos la orina mediante cistostomía suprapúbica.

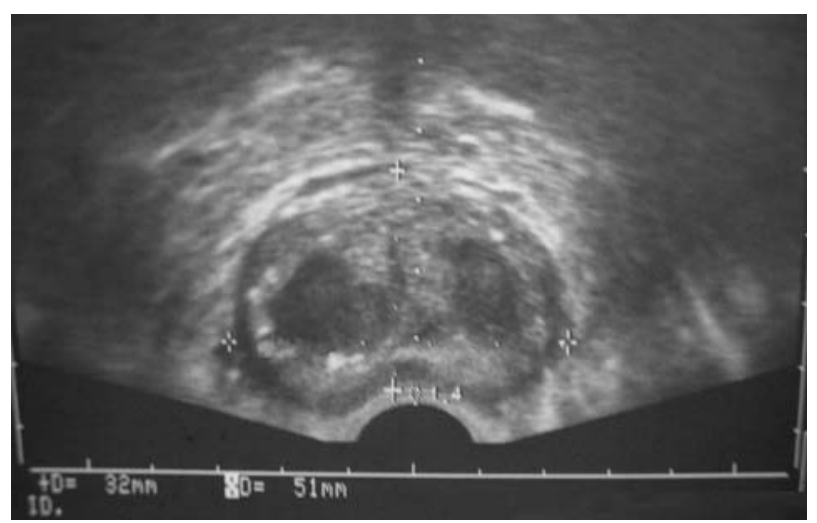

FIGURA 2: Imagen de ECTR en la que se aprecian dos zonas hipoecoicas de 28 y $30 \mathrm{~mm}$, sugerentes de absceso prostático, siendo el resto de la próstata de caracteristicas normales.

Del cultivo de las muestras (punción prostática y lumbar) se aisló S. aureus oxicilin sensible por lo que iniciamos tratamiento con Cloxacilina 2 g/24 y Rifampicina $300 \mathrm{mg} / 12 \mathrm{~h}$, y solicitamos una RMN lumbar urgente (Fig. 3) que se informó como empiema subdural, sin signos de espondilodiscitis.

El control posterior en base a nuevas ECTR se evidencia una franca mejoría, aún así es necesario drenar de nuevo un área hiperecogénica en el lóbulo prostático derecho, se evacuan 4cc de material purulento. Los controles posteriores no evidenciaron nuevas áreas sospechosas. Se realiza asimismo una ecografía transesofágica, que descarta endocarditis secundaria y una nueva RMN lumbar de control una vez completadas seis semanas de tratamiento antibiótico, que evidenció la total resolución del empiema subdural.

\section{DISCUSIÓN}

El absceso prostático es una entidad clínica poco frecuente La incidencia entre los pacientes con sintomatología del tracto urinario inferior oscila entre $0,5-2,5 \%{ }^{1,7}$.

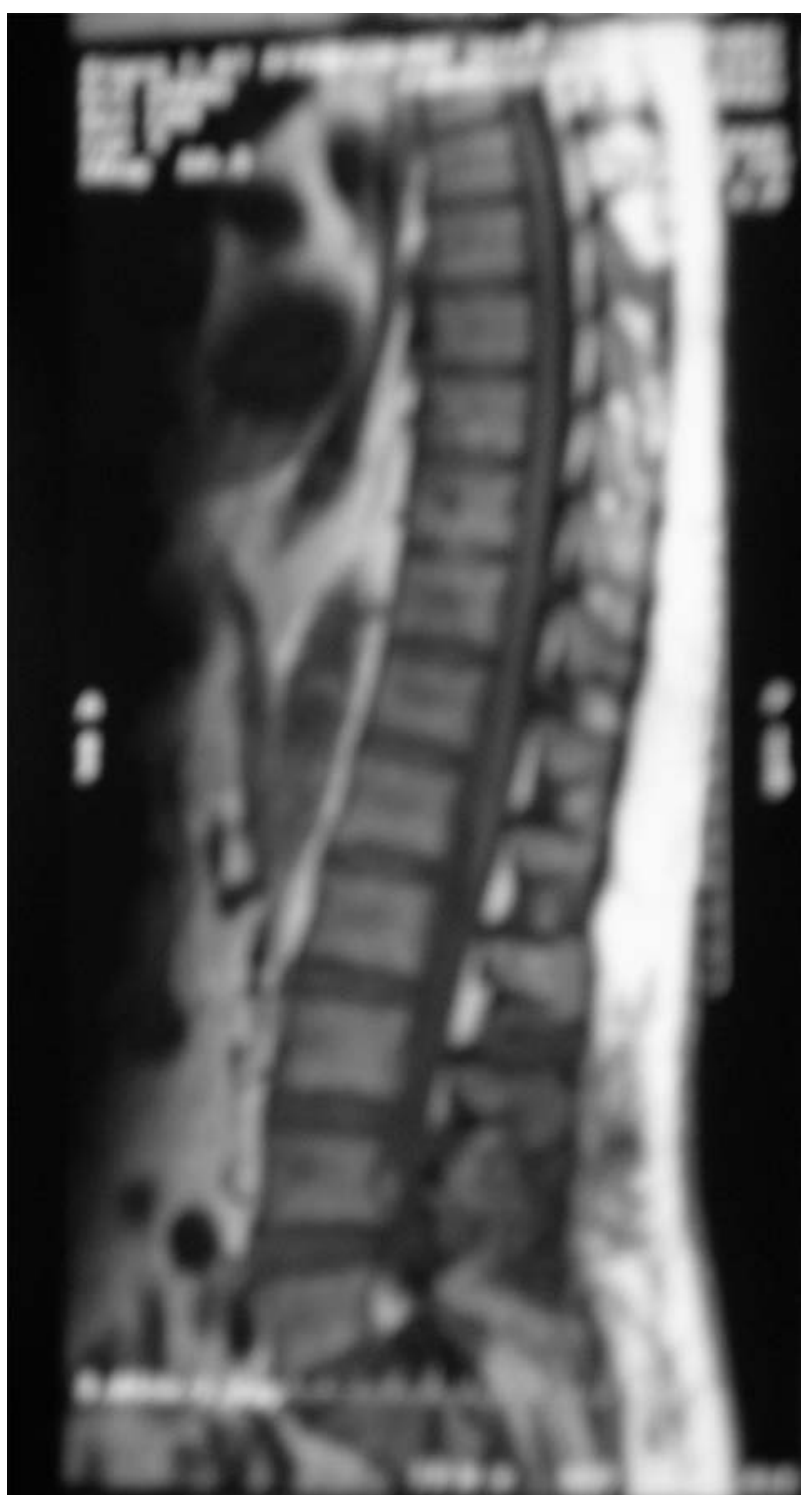

FIGURA 3: Imagen de RMN lumbar compatible con empiema subdural.

Existen dos mecanismos fisiopatológicos en la patogenia del absceso prostático, que definen a los grupos de riesgo ${ }^{13}$ :

- Pacientes con obstrucción del tracto urinario inferior y/o manipulación de la vía urinaria en los cuales, el reflujo de orina infectada es el mecanismo fundamental. En este caso el agente aislado con mayor frecuencia son los bacilos Gram negativos.

- Pacientes con mayor o menor grado de inmunosupresión (diabéticos, hepatópatas, y con ingresos hospitalarios frecuentes) y especialmente los adictos a drogas por vía parenteral en los 
cuales el Staphilococo aureus (oxacilin sensible y con menos frecuencia resistente) ocupa un lugar relevante. La diseminación hematógena es el mecanismo implicado.

Nuestro paciente pertenece a este segundo grupo, en particular en este caso, el Staphilococo se muestra como agente causante de abscesificación tras diseminación hematógena, aunque no sabemos la vía exacta de entrada.

Clínicamente la sintomatología local es la de una infección del tracto urinario inferior. El diagnóstico clínico es difícil por la inespecificidad de los sintomas ${ }^{3,8}$.Suele presentarse como un síndrome miccional, dolor perineal y ocasionalmente como retención aguda de orina ${ }^{9}$. Una intensa afectación del estado general y/o deterioro hemodinámico orientan al desarrollo de un proceso séptico. No debemos olvidar que la diseminación hematógena puede sobreañadir otros focos de afectación, en nuestro particular un dolor lumbosacro irradiado a ambos miembros inferiores debido a un empiema subdural.

El aspecto más importante de la exploración es el tacto rectal. Un tacto intensamente doloroso y la palpación de un foco fluctuante en la próstata obligan a practicar una exploración mediante pruebas de imagen. No obstante el tacto rectal es normal en la mayoría de las ocasiones, como era en nuestro paciente ${ }^{7,9,13}$.

La generalización en el uso de la ecografía transrectal por parte de los urólogos, ha convertido a esta técnica, en la de elección para el diagnóstico, con implicaciones además terapéuticas. La imagen típica es el de un área hipoecoica con ecos en su interior localizada en la zona central de una próstata difusamente aumentada de tamaño, que puede afectar a uno o ambos lóbu$\operatorname{los}^{4,7,10}$. La TAC y la RMN, se pueden emplear también en el diagnóstico.

En lo que respecta al tratamiento, dos son los pilares del tratamiento por un lado el tratamiento antibiótico y por otro lado el drenaje del absceso. La antibioterapia empírica debe cubrir a los microorganismos más frecuentemente causantes. La pauta empírica de nuestro hospital se realiza con una cefalosporina de tercera generación, aminoglucósidos y ampicilina ${ }^{13}$. En inmunodeprimidos y ADVP la cobertura debe incluir a los cocos Gram positivos, por lo que la vancomicina es de elección hasta conocer la sensibilidad a meticilina del microorganismo. Una vez aislado el microorganismo y determinada su sensibilidad antibioterápica, se modifica el tratamiento, que debe mantenerse durante al menos seis semanas.

En lo relativo al tratamiento quirúrgico las colecciones pequeñas $<1,5 \mathrm{~cm}$, pueden seguirse con ECTR de control administrándose tan solo tratamiento antibiótico y las colecciones de mayor tamaño deben drenarse ${ }^{7,10,11}$.

El drenaje quirúrgico de la colección se acepta como el factor más importante en el tratamiento del absceso prostático. Sin embargo, el método de drenaje es controvertido. Las técnicas de drenaje incluyen la incisión perineal y drenaje, la RTUP y el drenaje percutáneo transperineal ecodirigido mediante ECTR. Tradicionalmente se utilizaba la incisión perineal y drenaje de la colección, pero se ha ido abandonando debido al riesgo de impotencia que suponía, siendo utilizado hoy día para el drenaje de abscesos extraprostáticos ${ }^{8}$. La RTUP es en algunos centros la técnica de elección, pero no debemos olvidar que tiene una alta tasa de complicaciones como un mayor riesgo de bacteriemia por apertura de senos venosos, estenosis uretral y eyaculación retrógrada ${ }^{3,7}$.

$\mathrm{El}$ drenaje percutáneo transperineal ecodirigido mediante ECTR es un método seguro y eficaz en el drenaje de abscesos prostáticos. El mecanismo de esta técnica consiste en que al vaciar la colección se reduce la presión alrededor de la misma y de esta forma es más sencilla la entrada del antibiótico, a la cavidad drenada y a la próstata. Las tasas de éxito siguiendo este procedimiento en las series publicadas son del $83,3 \%{ }^{5,7,10}$. El abordaje transperineal se prefiere al transrectal ya que de esta forma se evita la formación de fistulas uretro-rectales y la contaminación con heces de la cavidad del absceso ${ }^{10,12}$.

\section{CONCLUSIONES}

El absceso prostático es hoy día una patología poco frecuente. Debido a la dificultad en el diagnóstico diferencial entre la prostatitis aguda y otras formas de infección urinaria en el varón y el absceso prostático, se necesita una alta sospecha clínica para el diagnóstico de esta entidad. Hacer un diagnóstico correcto es importante ya 
que el absceso prostático puede poner en peligro la vida de paciente y generar complicaciones graves. La realización de una ecografía transrectal esta indicada en pacientes con un tacto rectal sospechoso o en aquello con un cuadro de clínica compatible y evolución tórpida bajo cobertura antibiótica empírica en 48 horas. Este procedimiento ofrece el diagnóstico de certeza y la posibilidad de realizar una aspiración-drenaje ecodirigida de la colección, con escasa tasa de complicaciones asociada.

\section{REFERENCIAS}

1. Meares EM Jr: Prostatitis and related disorders. In Walsh PC, Retik AB, Stamey TA, et al (Eds): Campbell's Urology. $7^{\text {th }}$ Ed. Philadelphia, WB Saunders, 1992; p. 615.

2. Napal Lecumberri S, Verdú Martínez M, Monsalve Rodriguez M, Kilani Elsmari, S, Gómez Cisneros S.C, García Alonso J. Absceso prostático: nuevas guías diagnósticas y terapéuticas. Actas Urol Esp. 1992;16(2):144-147.

3. Jacobsen JD, Kvist E. Prostatic abscess: a review of literature an a presentation of five cases. Scand J Urol Nephrol 1993;27(2):281-284.

4. Sargent JC, Irwin R. Prostatic abscess clinical study of 42 cases. Am J Surg 1931;11:334-337.

5. Gogus C, Ozden E, Karaboga R, Yagci C. The value of transrectal ultrasound guided needle aspiration in treatment of prostatic abscess. Eur J Radiol 2004;52(1):94-98.

6. Franco A, MenÉndez V, Luque MP, Ribal MJ, Mallafre JM, Alcover $\mathrm{J}$ et al. Abscesos prostáticos: diagnóstico y tratamiento. Actas Urol Esp. 1996;20(2):189-192.

7. Collado A, Palou J, García-Penit J, José Salvador, Pablo de la Torre, JV. Ultrasound-guided needle aspiration in prostatic abscess. Urology 1999;53(3):548-552.
8. Gonzalez de Chaves E, Gomez de Segura C, Segarra J, Villavicencio Mavrich H. Prostatic abscess: report of a case. Arch Esp Urol. 2003;56(7):840-845.

9. Granados EA, Riley G, Salvador J, Vicente J. Prostatic abscess: diagnosis and treatment. J Urol 1992;148(1):80-82.

10. Lim JW, Ko YT, Lee DH, Park SJ, Oh JH, Yoon Y, Chang SG. Treatment of prostatic abscess: value of transrectal ultrasonographically guided needle aspiration. J Ultrasound Med 2000;19(9):609-617.

11. Bosquet Sanz M, Gimeno Argente V, Palmero Marti JL, Bonillo García JV, Salom Fuster JF, Jimenez Cruz. Absceso prostático: revision de la literatura y presentación de un caso. Actas Urol Esp 2005;29:100-104.

11. Bosquet Sanz M, Gimeno Argente V, Palmero Marti JL, Bonillo García JV, Salom Fuster JF, Jimenez Cruz. Prostatic abscess: report of a case and review of the literature. Actas Urol Esp 2005;29:100-104.

12. Ludwing M, Schoroeder-Printzen I, Schiefer HG, Wolfgang Weidner. Diagnosis and therapeutic management of 18 patients with prostatic abscess. Urology 1999;53(2):340-345.

13. Millán Rodríguez F, Orsola de los Santos A, Vayreda Martija JM, Chechile Toniolo G. Manejo de las prostatitis agudas: experiencia con 84 pacientes. Arch Esp Urol. 1995;48(2):129-136.

13. Millán Rodríguez F, Orsola de los Santos A, Vayreda Martija JM, Chechile Toniolo G. Management of acute prostatitis: experience with 84 patients. Arch Esp Urol. 1995;48(2):129-136.

Correspondencia autor: Dr. F. Cabrera Meirás.

Servicio de Urolgoía. Hospital Universitario 12 de Octubre. Avda. de Cordoba s/n - 28041 Madrid.

Tel.: 913908121

E-mail autor: cabdoc@hotmail.com

Información artículo: Nota clínica

Trabajo recibido: marzo 2006

Trabajo aceptado: abril 2006 